\title{
Geometric Topics on Elementary Amenable Groups
}

\author{
Mostafa Ftouhi*, Mohammed Barmaki, Driss Gretete
}

Ecole nationale des sciences appliquées Université Ibn Tofail campus universitaire, Morocco

Received November 1, 2019; Revised November 23, 2019; Accepted November 30, 2019

Copyright (C) 2020 by authors, all rights reserved. Authors agree that this article remains permanently open access under the terms of the Creative Commons Attribution License 4.0 International License

Abstract The class of amenable groups plays an important role in many areas of mathematics such as ergodic theory, harmonic analysis, representation theory, dynamical systems, geometric group theory, probability theory and statistics. The class of amenable groups contains in particular all finite groups, all abelian groups and, more generally, all solvable groups. It is closed under the operations of taking subgroups, taking quotients, taking extensions, and taking inductive limits. In 1959, Harry Kesten proved that there is a relation between the amenability and the estimates of symmetric random walk on finitely generated groups. In this article we study the classification of locally compact compactly generated groups according to return probability to the origin. Our aim is to compare several geometric classes of groups. The central tool in this comparison is the return probability on locally compact groups. we introduce several classes of groups in order to characterize the geometry of locally compact groups compactly generated. Our aim is to compare these classes in order to better understand the geometry of such groups by referring to the behavior of random walks on these groups. As results, we have found inclusion relationships between these defined classes and we have given counterexamples for reciprocal inclusions.

Keywords Graphs and Groups; Subgroup Growth;Wreath
Product; Probability Measures on Groups; Geometric Probability, Random Walks

\section{AMS}

Classification

Numbers:

\section{Introduction}

The class of amenable groups was introduced by von Neumann in 1929 (see [1]) in order to explain the HausdorffBanach-Tarski paradox [2].

Since then, the theory of amenable groups has advanced on many fronts. Recall that a topological group is said to be amenable if it admits an invariant continuous means, that is a functional $m$ on $L^{\infty}(G)$ such that $m(1)=1$ and for all $g \in G$ and for all $f \in L^{\infty}(G), m(g \cdot f)=m(f)$, where $g \cdot f(x)=f\left(g^{-1} x\right)$.

It is well known that finite groups and abelian groups are amenable and that the class $A G$ of amenable groups is closed under four standard processes of constructing new groups from given ones:

(A) Taking closed subgroups.

(B) Taking quotient groups. 
(C) Group extensions.

(D) Direct limits.

In the sequel, we introduce several classes of groups in order to characterize the geometry of locally compact groups compactly generated. Our aim is to compare these classes in order to better understand the geometry of such groups by referring to the behavior of random walks on these groups.

\section{Word distance and geometric proper- ties}

Let $G$ be a locally compact group and compactly generated with identity element $e$. Let $\lambda$ be the Haar measure on $G$. For any compact generating set $\mathrm{K}$, symmetric and neighborhood of $e$, consider the Cayley graph $(G, K)$ with vertex set $G$ and an edge from $x$ to $y$ if and only if $y=x z$ for some $z \in K$.

For all $x \in G$, the length $l_{K}(x)$ of $x$ associated to $K$ is the minimal number $n$ of elements $s_{1}, \ldots, s_{n} \in K$ such that $x=s_{1} s_{2} \ldots s_{n}$, we put by convention $l_{K}(e)=0$.

We denote by $d_{K}(x, y)=l_{K}\left(x^{-1} y\right)$ the word distance between the elements $x, y$ in the Cayley graph $(G, K)$.

The volume growth function on $G$ associated to the compact $K$ is defined by $V_{K}(n)=\lambda\left(B_{K}(n)\right)$, where $B_{K}(n)$ is the ball centered at $e$ and with radius $n$ with respect to the word distance.

We define the asymptotic behavior of the volume growth in the following sense :

if $f$ and $g$ are two non-negative functions defined on the positive real axis, we use the notation $f \lesssim g$ if there exist constants $a, b>0$, such that for $\mathrm{x}$ large enough, $f(x) \leq a g(b x)$. If the symmetric relation also holds, we write $f \simeq g$.

When a function is defined only on the integers, we extend it to the positive real axis by linear interpolation. We will use the same name for the original function and its extension. If $f \lesssim g$ holds without $f \simeq g$, we write $f \lesssim g$.

The asymptotic behavior of $f$ is the coset with respect to this relation.

It is well known that the asymptotic behavior of $V_{K}(n)$ is independent of the choice of $K$. So, we can denote it in the sequel by $V_{n}$.

For a group $G$, three behaviors may occur:

- Exponential volume growth when $V_{n} \simeq \exp (n)$,

- Polynomial volume growth when $V_{n} \simeq n^{d}$ for some $d>0$

- Intermediate growth, where $V_{n}$ is equivalent to neither of the above.

It is obvious that $\lim _{n}\left(V_{n}\right)^{1 / n}$ exists for all locally compact group $G$ compactly generated. If this limit is strictly greater than 1 , the group has exponential growth. If it is at most 1 , we say that $G$ has sub-exponential growth.

\section{Probability of return to the origin}

Let $G$ be a locally compact, compactly generated and unimodular group.

Let $e$ be the unit element of $G$. Let $\lambda$ be the Haar measure on $G$. Let $\mu$ be a probability measure on $G$ associated to a density $F$ with respect to $\lambda$.

We suppose that $F$ satisfies the following "natural assumptions":

1. $F$ is bounded

2. $F$ is symmetric

3. $F$ is locally positive, that is there exists a relatively compact symmetric open neighborhood $U$ of $e$ generating $G$, such that, for all $g \in U, F(g)>C$ for a positive constant C.
4. $F$ has a second finite moment, that is $\int_{G} l_{K}(g)^{2} F(g) d \lambda(g)<+\infty$.

We consider the universe $\Omega=G^{\mathbb{N}}$ equipped with the product Borelian $\sigma$-algebra denoted by $\mathbb{B}(\Omega)$. We define the probability space $(\Omega, \mathbb{B}(\Omega), P)$ by setting:

$$
P=\delta_{e} \otimes \mu^{\otimes \mathbb{N}},
$$

where $\delta_{e}$ is the Dirac measure at $e$. 
Let $X_{n}: \Omega \rightarrow G$ be the $n-t h$ canonical projection on $G$, with $X_{0}$ the sure variable equal to $e$, and $Z_{n}(\omega)=$ $\prod_{i=0}^{n} X_{i}(\omega) ; \omega \in \Omega$ defines as in [3] the random walk on $G$ associated to $\mu$.

It is well known that the asymptotic behavior of the probability $P\left(Z_{2 n} \in U\right)$ is independent of the choice of $F$, and $P\left(Z_{2 n} \in U\right) \simeq F^{* 2 n}(e)$, where $F^{* 2 n}$ is the $2 n-t h$ convolution of $F$ by itself. In the rest we denote by $\Phi_{G}(n)$ the asymptotic behavior of $P\left(Z_{2 n} \in U\right)$ called the asymptotic decay of the return probability to the origin on the group $G$.

\section{Comparison between Classes in a dis- crete case}

In this section, we consider the following classes of finitely generated discrete groups.

1. The class $A G$ of all amenable groups.

2. The class $E G$ which is the smallest class containing finite groups, abelian groups, and closed under processes: (A)(D).

3. The class $N F$ of groups without free subgroup on two generators.

4. The class $I G$ of groups with asymptotic behavior of probability of return decays slower than $\exp \left(-n^{1 / 3}\right)$, that is $\Phi_{G}(n) \gtrsim \exp \left(-n^{1 / 3}\right)$.

5. The class $S G$ : the smallest class containing all groups with sub-exponential volume growth and closed under processes: (A)-(D).

The notations $A G$ and $N F$ were also introduced by Day [4] in the context of discrete groups. We will call groups in $E G$ elementary amenable groups.

In the class $S G$ that was introduced by Rosenblatt [5], the groups are said to be sup-amenable.

In this paper, we give a new description of the known amenable groups. More precisely, we will discuss the relation- ship between the classes above. The tool used in this comparison is the asymptotic decay of $\Phi_{G}(n)$.

\subsection{Comparison between EG, SG and AG}

Proposition 1. (see [6, 7])

The class EG is a subset of $S G$, the converse is false.

Proof.

If $G$ is abelian or finite, then by the Gromov's Growth theorem ( see [8]) $G$ must have a polynomial volume growth of some degree $d$, that is $V_{G}(n) \simeq n^{d}$ so $V_{G}(n)^{1 / n} \rightarrow 1$, then $G \in S G$.

Hence, $S G$ contains every abelian and finite groups, and since it is closed under processes (A)-(D) so $E G \subseteq S G$.

The first Grigorchuk group is with intermediate volume growth. So by Chou's theorem $G_{\text {first }}$ is not in $E G$. On the other hand, $G_{\text {first }}$ has a sub-exponential volume growth. That proves that $G_{\text {first }}$ is in $S G$, so $S G$ is not a subclass of $E G$. (For a detailed definition of the $G_{\text {first }}$ group, see [9]).

\section{Proposition 2. (see [10])}

The class $S G$ is a subset of $A G$, the converse is false.

Proof.

Let $G$ be a group with a sub-exponential volume growth. If $G$ is not amenable, then by Kesten's theorem, $\Phi_{G}(n) \simeq$ $\exp (-n)$, and taking $K$ such that $\operatorname{supp}(F) \subset K$ then $P\left(Z_{2 n} \in B_{2 n}\right)=1$. On the other hand, using Cauchy Schwarz inequality, we get $P\left(Z_{2 n} \in B_{2 n}\right) \leq F^{* 2 n}(e) \lambda\left(B_{2 n}\right)$ so $\lambda\left(B_{2 n}\right) \geq \frac{1}{F^{* 2 n}(e)}$ and then $\exp (n) \lesssim V_{G}(n)$. That gives $G \in A G$. Since $A G$ is closed under (A)-(D), we get the desired inclusion.

Consider the iterated monodromy group $G$ of the polynomial $z^{2}-1$, that was introoduced by Grigorchuk and Zuk in [7], who showed that $G$ does not belong to the class $S G$.

In [10], Bartholdi and Balint Virag showed that $\Phi_{G}(n) \simeq$ $\exp \left(-n^{2 / 3}\right)$ and so by Kesten's criterion $G$ is amenable. This shows that the inclusion $S G \subseteq A G$ is strict. 


\subsection{Comparison between AG and NF}

Proposition 3. (see [10])

The class $A G$ is a subset of NF, the converse is false.

\section{Proof.}

Let $G$ be a group which is not in $N F$. Then, it contains a free group $F$ with two generators. Since $G$ is discrete, so $F$ is a closed subset of $G$ and using Theorem 1.3 in [11] $\Phi_{G}(n) \lesssim$ $\Phi_{F}(n)$. It is well known that $\Phi_{F}(n) \simeq \exp (-n)$, so $\Phi_{G}(n) \lesssim$ $\exp (-n)$. Then by Kesten's theorem $G$ is not amenable.

In the converse problem that was solved in the 1980's by Ol'shanskii [12], he proved that $A G \neq N F$ by showing that the Tarski monster group, which is an infinite group in which every nontrivial proper subgroup is cyclic of order and a fixed prime $p$, is not amenable and it does not contain a free nonabelian group with two generators.

\subsection{Comparison between AG and IG}

Proposition 4. The class $I G$ is a subset of $A G$, the converse is false.

Proof.

Let $G$ be in $I G$ then $\Phi_{G}(n) \gtrsim \exp \left(-n^{1 / 3}\right)$ so $\exp (-n) \nsim$ $\Phi_{G}(n)$. Then by Kesten's theorem, the amenability of $G$ holds. To show that converse is not true, we use the iterated wreath product $(\mathbb{Z} \neg \mathbb{Z}) \prec \mathbb{Z}$ then by [13] we have

$$
\Phi(n) \simeq \exp \left(-n^{1 / 2} \log ^{1 / 2} n\right)
$$

then $(\mathbb{Z} \imath \mathbb{Z}) \prec \mathbb{Z}$ is in $A G$ but not in $I G$.

\subsection{Comparison between EG and IG}

Proposition 5. The class EG is a subset of $I G$.

Proof.

Suppose that $G$ is in $E G$. Then by Chou's theorem [6]; $G$ has either polynomial or exponential volume growth.

- In the polynomial case, $\Phi_{G}(n) \simeq n^{-d / 2}$ so $G$ in $I G$.

- In the exponential case, by Corollary 7.4 in [14] the group $G$ satisfies $\Phi_{G}(n) \simeq \exp \left(-n^{1 / 3}\right)$ and then $G$ is also in $I G$.
Question: Is it true that $I G$ is not a subset of $E G$ ?

\section{Summary}

We summarize the results obtained in the following diagram

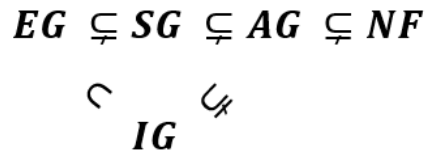

Such as :

- $A G$ : The class of all amenable groups.

- $E G$ : The class which is the smallest class containing finite groups, abelian groups, and closed under processes (A)-(D).

- $N F$ : The class of groups without free subgroup on two generators

- $I G$ : The class of groups with asymptotic behavior of probability of return decays slower than $\exp \left(-n^{1 / 3}\right)$, that is $\Phi_{G}(n) \gtrsim \exp \left(-n^{1 / 3}\right)$.

- $S G$ : The smallest class containing all groups with subexponential volume growth and closed under processes (A)-(D).

\section{Funding}

This research received no external funding.

\section{Conflicts of Interest}

The authors declare no conflict of interest. 


\section{REFERENCES}

[1] J.von Neumann, Zur allgemeinen Theorie des Masses, Fundamenta Mathematicae. 13 (1929), Issue: 1, 73-116.

[2] St. Banach and A. Tarski, Sur la décomposition des ensembles de points en parties respectivement congruentes, Fund. math. 6 (1924), 244-277.

[3] Gretete, D. Stabilité du comportement des marches aléatoires sur un groupe localement compact, Ann. Inst. Henri Poincaré Probab. Stat. 44(2008), no. 1, 129-142.

[4] M.M. Day, Amenable semigroups, Illinois J. Math., 1 (1957), 509-544.

[5] J. Rosenblatt, Invariant measures and growth conditions, Trans. Amer. Math. Soc. 193 (1974), 33-53.

[6] C. Chou (1980). Elementary amenable groups. Illinois J. Math., 24(3):396-407.

[7] R .I. Grigorchuk and A. Zuk (2002). On a torsion-free weakly branch group defined by a three state automaton. Internat. J. Algebra Comput., 12(1-2):223-246. International Conference on
Geometric and Combinatorial Methods in Group Theory and Semigroup Theory (Lincoln, NE, 2000).

[8] Gromov, M.: Groups of polynomial growth and expanding maps. Inst. Hautes Études Sci. Publ. Math. 53, 53-73 (1981)

[9] R. I. Grigorchuk (1998). An example of a finitely presented amenable group that does not belong to the class EG. Mat. Sb., 189(1):79-100.

[10] Laurent Bartholdi and Balint Virag, Amenability via random walks. Duke Math. J. 130 (2005), no. 1, 39-56.

[11] C. Pittet. and L. Saloff-Coste, On the stability of the behavior of random walks on groups. J. Geom. Anal. 10 713-737.(2000)

[12] A.Yu. Ol'shanskii. An infinite group with subgroups of prime orders. Math. USSR, Izv., 16:279-289, 1981.

[13] Pittet, C. and Saloff-Coste, L. (2002). On random walks on wreath products. Ann. Probab. 30 948-977.

[14] Coulhon, Th., Grigor'yan, A., Pittet, Ch.: A geometric approach to on-diagonal heat kernels lower bounds on groups. Ann. Inst. Fourier 51, 1763-1827 (2001). 